\title{
The olfactory receptor database: web-based resources for the genomics, proteomics and function of olfactory receptors
}

\author{
Chiquito J Crasto
}

\author{
From 1st International Workshop on Odor Spaces \\ Hannover, Germany. 4-7 September 2013
}

Olfactory receptors, the largest family in the genome, present an enormous challenge to understanding the molecular basis of how the information contained in odor molecules is transformed into the neural basis of smell perception. The Olfactory Receptor Database (ORDB) was created to support research with this goal. This talk will summarize salient aspects of ORDB as a comprehensive repository that stores and disseminates information related to the genomic, proteomics and functional aspects of olfactory receptors. ORDB is the result of almost two decades of work that has combined the efforts of chemosensory receptor experts, neuroscientists, computational experts, informatics researchers and clinicians, plus a growing community of contributors. Currently containing close to 15,000 olfactory and other chemosensory receptors, ORDB represents the work of more than a 100 laboratories worldwide, and contains receptors information for more than a hundred species. ORDB garners several hundred thousand visits from all over the world every year.

ORDB is designed as a web-interface to a database architecture that makes for efficient storage and dissemination of the knowledge. More importantly, it allows the integration of heterogeneous databases within its framework-through a metadata-driven infrastructure. Two companion databases to ORDB-OdorDB (a repository of odor molecule with emphasis on those known to have been experimentally shown to activate ORs) and the new OdorModelDB (a repository of the results of computational modeling experiments on ORs) will also be described. Together, these three component databases aim to provide investigators with a comprehensive framework for understanding odor ligand-olfactory receptor interactions as the initial step in the neural processing of odor information. A fourth component, OdorMapDB, is a resource for investigating how the ligand-receptor interactions lead to representation as spatial activity maps in the olfactory bulb as the nextl step toward the neural basis of smell perception.

Also discussed in this presentation will be efforts towards the development of software that not only populates ORDB in an automated manner, but also automatically updates data for relevance and timeliness.

The unique infrastructure on which the olfactory databases are based is part of the SenseLab suite of databases. This infrastructure allows the resources to be searched for entries using a multi-parameter search system. These parameters are descriptors of various instances of information stored in ORDB. Such a search system allows the return of few specific and relevant results from several thousand.

A live web-demonstration of ORDB, OdorDB and OdorModeDb will be part of the presentation.

Published: 16 April 2014

doi:10.1186/2044-7248-3-S1-O8

Cite this article as: Crasto: The olfactory receptor database: web-based resources for the genomics, proteomics and function of olfactory receptors. Flavour 2014 3(Suppl 1):O8. 